\section{Normatividad que rige la investigación clínica en seres humanos y requisitos que debe cumplir un centro de investigación para participar en un estudio clínico en México}

López-Pacheco $\mathrm{MC}^{1^{*}}$, Pimentel-Hernández $\mathrm{C}^{2 *}$, Rivas-Mirelles $\mathrm{E}^{2}$, ArredondoGarcía JL2*

Resumen

Revisión de los principios científicos, éticos y regulatorios nacionales e internacionales aplicables para conducir estudios clínicos en México, con especial interés en las atribuciones de regulación, control y fomento sanitarios conforme a la Ley General de Salud y a través de la Comisión Federal para la Protección contra Riesgos Sanitarios. Se recopilan los pasos y requisitos que deben cumplir un investigador principal y un centro de investigación para participar en la conducción de estudios clínicos multicéntricos, multirregionales, con financiamientos federales o privados.

PALABRAS CLAVE: estudio clínico, ética, Buenas Prácticas Clínicas, Ley General de Salud (LGS), investigador principal, centro de investigación.

Acta Pediatr Mex. 2016 May;37(3):175-182.

\section{Regulations governing humans' clinical research and requirements to be met by a research center to participate in a clinical trial in Mexico.}

López-Pacheco $\mathrm{MC}^{1^{*}}$, Pimentel-Hernández $\mathrm{C}^{2 *}$, Rivas-Mirelles $\mathrm{E}^{2}$, ArredondoGarcía JL2*

\section{Abstract}

We reviewed national and international scientific, ethical and regulatory principles needed to conduct clinical research in Mexico, with emphasis on the capability that has the General Health Law, trough the Federal Commission for the Protection against Sanitary Risk to regulate, control, and promote health. We colected steps and requirements that need to be accomplished by the principal investigator and research center to participate in the conduction of multicenter and multiregional clinical research studies, with federal or private funding.

KEYWORDS: clinical research; ethics; Good Clinical Practices; COFEPRIS; General Health Law; principal investigator; research center
${ }^{1} \mathrm{PhD}$. Biomedicina y Biotecnología Molecular.

${ }^{2}$ Médico Pediatra.

*Subdirección de Investigación.

Instituto Nacional de Pediatría.

Recibido: 5 de marzo del 2016

Aceptado: 22 de abril del 2016

Correspondencia

Dr. José Luis Arredondo García

Subdirector de Investigación Médica

Instituto Nacional de Pediatría

Insurgentes Sur 3700-C

04530 Ciudad de México

Tel. 10840900 ext. 1137-1901

joselag@unam.mx

arredondo.joseluis@gmail.com

Este artículo debe citarse como

López-Pacheco MC, Pimentel-Hernández C, RivasMirelles E, Arredondo-García JL. Normatividad que rige la investigación clínica en seres humanos y requisitos que debe cumplir un centro de investigación para participar en un estudio clínico en México. Acta Pediatr Mex 2016;37(3):175-182. 


\section{INTRODUCCIÓN}

El desarrollo de la investigación para la salud debe atender aspectos éticos y científicos que garanticen la dignidad y el bienestar de la persona sujeta a investigación; requiere del establecimiento de criterios técnicos y lineamientos claros para regular la aplicación de los procedimientos relativos a la correcta utilización de los recursos destinados a ella. Es por ello que en este documento se revisan los fundamentos y pasos que debe seguir cualquier centro de investigación e investigador principal para poder ser candidatos a conducir estudios clínicos en México, en cualquier fase de investigación, multicéntrico y multirregión, con financiamiento federal o privado. Comienza con la revisión de la normatividad legal nacional e internacional actual que rige la investigación clínica; se definen conceptos importantes y las responsabilidades que adquirirá el investigador principal al participar en estudios clínicos; se enlistan los requisitos que debe cumplir el centro de investigación e investigador principal para obtener la aprobación del comité de ética en investigación y de COFEPRIS; se resumen algunos cometidos que debe efectuar el investigador principal una vez obtenida la aprobación para conducir un estudio clínico y los productos que obtienen de su participación.

\section{Objetivo}

Proporcionar al lector un artículo referente con respecto a los conocimientos científicos, éticos y regulatorios que rigen los estudios clínicos en México, desde un punto de vista crítico, actual y completo.

\section{Normatividad legal que rige la Investigación clínica internacional y en México}

Para la realización de cualquier estudio clínico, sin importar la fase de investigación en la que se encuentre, ya sea para el empleo de insumos para la salud, procedimientos o actividades experimen- tales en seres humanos o muestras biológicas de seres humanos, deben respetarse los principios científicos, éticos y regulatorios nacional e internacionalmente aceptados. Estos son: el Código de Núremberg (1947); ${ }^{1}$ la Declaración de Helsinki $(1964)^{2}$ y sus enmiendas de Tokio(1975), ${ }^{3}$ Venecia (1983), ${ }^{4}$ Hong Kong (1989), ${ }^{5}$ Somerset West, Sudáfrica $(1996)^{6}$ y Edimburgo (2000); ${ }^{7}$ las Guías Éticas Internacionales para Investigación Biomédica que Involucra a Seres Humanos del Consejo de Organizaciones Internacionales de la Ciencias Médicas $(\mathrm{ClOMS})^{8}$ y las Buenas Prácticas Clínicas: documentos de las Américas de la Organización Panamericana de la Salud como oficina regional de la Organización Mundial de la Salud (OMS); ${ }^{9}$ las Guías para la Buenas Prácticas Clínicas de la Conferencia Internacional de Armonización (ICH, E6-R1);i1 así como La Ley General de Salud $(\mathrm{LGS})^{11}$ en México a través del Reglamento de la Ley General de Salud en Materia de Investigación para la Salud; ${ }^{12}$ el Reglamento de la Comisión Federal para la Protección contra Riesgos Sanitarios; ${ }^{13}$ las Normas Oficiales Mexicanas, Acuerdos y Guías en la materia publicadas en el Diario Oficial de la Federación, donde se han establecido los lineamientos, trámites y principios a los cuales deberá someterse la investigación para la salud en México para contar con autorización de la Comisión Federal para la Protección contra Riesgos Sanitarios (COFEPRIS). ${ }^{14}$ El cumplimiento de estas guías asegura la protección de la dignidad, derechos humanos, seguridad y bienestar de los participantes en el estudio clínico.

La COFEPRIS establece las atribuciones en materia de regulación, control y fomento sanitarios y cuenta con una unidad administrativa denominada Comisión de Autorización Sanitaria, responsable de proponer los requisitos y disposiciones administrativas para la operación de establecimientos destinados a procedimientos o actividades clínicas o de investigación y se encarga de expedir, prorrogar o revocar la autorización de proyectos para el empleo de medicamentos, materiales, aparatos, procedi- 
mientos o actividades experimentales en seres humanos con fines de investigación científica, respecto de los cuales no se tenga evidencia científica suficiente para probar su eficacia preventiva, terapéutica o de rehabilitación. ${ }^{14}$

La Ley General de Salud, título quinto "Investigación para la Salud", capítulo único, establecen los lineamientos y principios a los cuales debe someterse la investigación en materia de salud que involucre seres humanos o muestras biológicas derivadas de estos; ${ }^{11}$ de este título deriva el Reglamento de la Ley General de Salud en Materia de Investigación para la Salud, el cual establece que la investigación en materia de salud es un factor determinante para mejorar las acciones encaminadas a proteger, promover y restaurar la salud del individuo y de la sociedad en general; para desarrollar tecnología mexicana en los servicios de salud, para incrementar su productividad y para efectuar actividades de formación y desarrollo de personal para la salud. ${ }^{12}$

El artículo 100 de la Ley General de Salud establece que la investigación en seres humanos deberá adaptarse a los principios científicos y éticos que justifiquen la investigación médica; que sea el único método por el cual se pueda obtener este conocimiento; que se asegure que el sujeto de experimentación no se expone a riesgos ni daños innecesarios; se cuente con el consentimiento informado por escrito del sujeto de investigación o de su representante legal y se realice únicamente por profesionales de la salud en instituciones médicas autorizadas y vigiladas por las autoridades sanitarias correspondientes. En toda investigación en la que el ser humano sea sujeto de estudio deberá prevalecer el criterio del respeto a su dignidad, protección de sus derechos y bienestar sobre cualquier otro interés de la ciencia y la sociedad. ${ }^{11}$

El título sexto de la Ley General de Salud en Materia de Investigación corresponde a la ejecución de la investigación en instituciones de atención a la salud, engloba las características, funciones, obligaciones y/o responsabilidades que debe poseer el investigador principal que estará a cargo de la investigación, como la presentación de un informe técnico ante el Comité de Ética en Investigación de la institución de atención a la salud; la publicación de informes debe de mantener la confidencialidad de los sujetos de investigación, así como la que se haya acordado con los patrocinadores del estudio. Además, se debe dar crédito a los investigadores asociados y al personal técnico participante y entregar una copia de las publicaciones a la dirección de la institución ${ }^{12}$ (Figura 1).

\section{Definición y responsabilidades del investigador principal/centro de investigación en la conducción de estudios clínicos de acuerdo con la normativa legal mexicana}

Estos lineamientos establecen que un centro de investigación es aquel establecimiento de atención médica perteneciente a los sectores público, social o privado, cualquiera que sea su denomina-

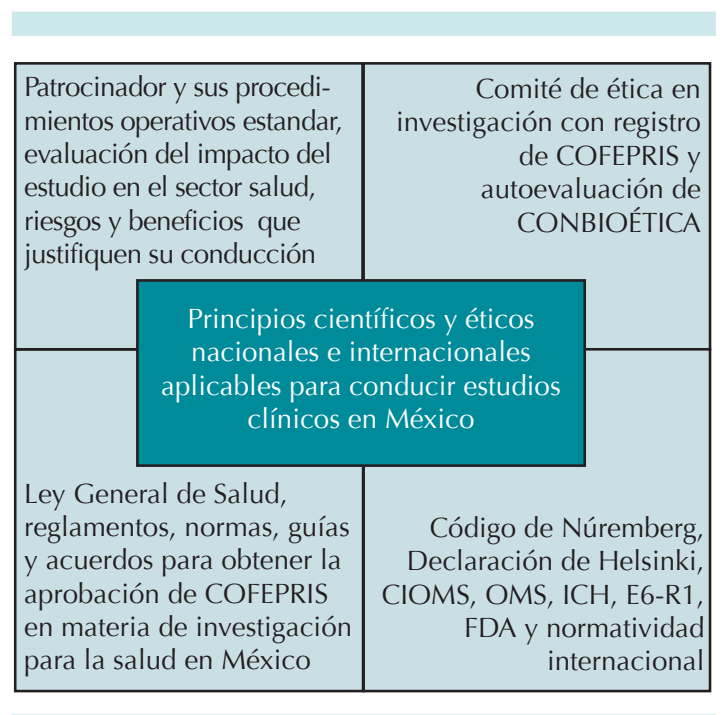

Figura 1. Principios científicos y éticos nacional e internacionalmente aplicables para conducir estudios clínicos en México. 
ción, que pueda efectuar actividades preventivas, diagnósticas, terapéuticas, de rehabilitación, así como los terceros autorizados para realizar los estudios de intercambiabilidad de medicamentos, dirigidas a mantener o reintegrar el estado de salud de las personas y efectuar actividades de formación y desarrollo de personal para la salud, así como de investigación en seres humanos y/o sus productos; y que de conformidad con la Ley General de Salud, Reglamentos y demás disposiciones aplicables, están obligados a contar con un Comité de Ética en Investigación. ${ }^{15}$

El investigador principal es el profesional de la salud que es responsable de conducir, coordinar, dirigir y vigilar la ejecución de una investigación para la salud en seres humanos y que cuenta con la formación académica y experiencia adecuada para ello. Tiene la obligación de conocer y aplicar lo establecido en la Ley General de Salud, sus reglamentos y en las Buenas Prácticas Clínicas (ICH-E6-R1) antes, durante y después de la conducción de la investigación, incluyendo la confidencialidad del proyecto, así como el apego al protocolo clínico. Debe proteger la vida, dignidad, integridad, derecho, privacidad, proporcionar asistencia médica y salvaguardar la salud de los sujetos que participen en la investigación propuesta; aplicando su conocimiento y ética para cumplir con este deber sobre cualquier otro interés. Tiene la responsabilidad de seleccionar a su equipo de trabajo, quienes deben tener el conocimiento, educación y entrenamiento en Buenas Prácticas Clínicas (ICH-E6- R1) y en el proceso de la investigación en que esté involucrado cada individuo, así como la conducción del proceso de obtención del consentimiento informado, debe de entregar al sujeto en investigación la información de contacto (teléfono de 24 horas, correo, nombre del contacto) para los casos de emergencia. Debe notificar a la COFEPRIS el estatus del reclutamiento de los sujetos, indicando al menos la fecha de reclutamiento del primer sujeto e informar semestralmente el estatus del reclutamiento. Deberá conservar todo convenio, contrato o registro en donde se estipulen las responsabilidades, atribuciones y funciones de todos los involucrados en la investigación; tiene la responsabilidad de reportar y garantizar la calidad y validez de los datos obtenidos durante la investigación. ${ }^{15}$

El investigador principal tiene además la responsabilidad de reportar todo evento adverso derivado de la investigación de acuerdo con lo establecido en la NOM-220-SSA1-2012 "Instalación y operación de la farmacovigilancia,"16 así como la responsabilidad de elaborar, integrar, utilizar, archivar y asegurar el resguardo del expediente clínico del sujeto en investigación de acuerdo con lo establecido en la NOM-168-SSA1-1998, "del Expediente Clínico" así como en las Buenas Prácticas Clínicas (ICH-E6-R1) y las Buenas Prácticas de Documentación (BPD); entendiéndose por tiempo mínimo de resguardo 5 años de acuerdo con la NOM-168-SSA1-1998. $15,17,18$

La NOM-004-SSA3-2012 del Expediente clínico establece los criterios científicos, éticos, tecnológicos y administrativos obligatorios en la elaboración, integración, uso, manejo, archivo, conservación, propiedad, titularidad y confidencialidad del expediente clínico, el cual se constituye en una herramienta de uso obligatorio para el personal del área de la salud, de los sectores público, social y privado que integran el Sistema Nacional de Salud. ${ }^{19}$

La NOM-012-SSA3-2012 establece los criterios para la ejecución de proyectos de investigación para la salud en seres humanos. ${ }^{20}$

El investigador no deberá implementar ninguna desviación o cambios al protocolo sin previo acuerdo del patrocinador y una revisión y aprobación favorable del Comité de Ética en Investigación, excepto en caso de peligro inmediato a los sujetos del estudio. Presentará al Comité de Ética en Investigación resúmenes escritos del estatus del estudio en forma anual 
o con mayor frecuencia si así se solicita. Si el estudio se termina o suspende prematuramente por cualquier motivo, el investigador deberá informar rápidamente a los sujetos del estudio, asegurar un tratamiento y un seguimiento apropiado para estos e informar al Comité de Ética en Investigación y COFEPRIS ${ }^{15}$ (Figura 2).

\section{Requisitos para que el investigador principal/} centro de investigación obtenga el dictamen favorable para la conducción del estudio clínico

Antes de iniciar cualquier estudio el investigador debe contar con la aprobación favorable, escrita y fechada por parte del Comité de Ética en In-

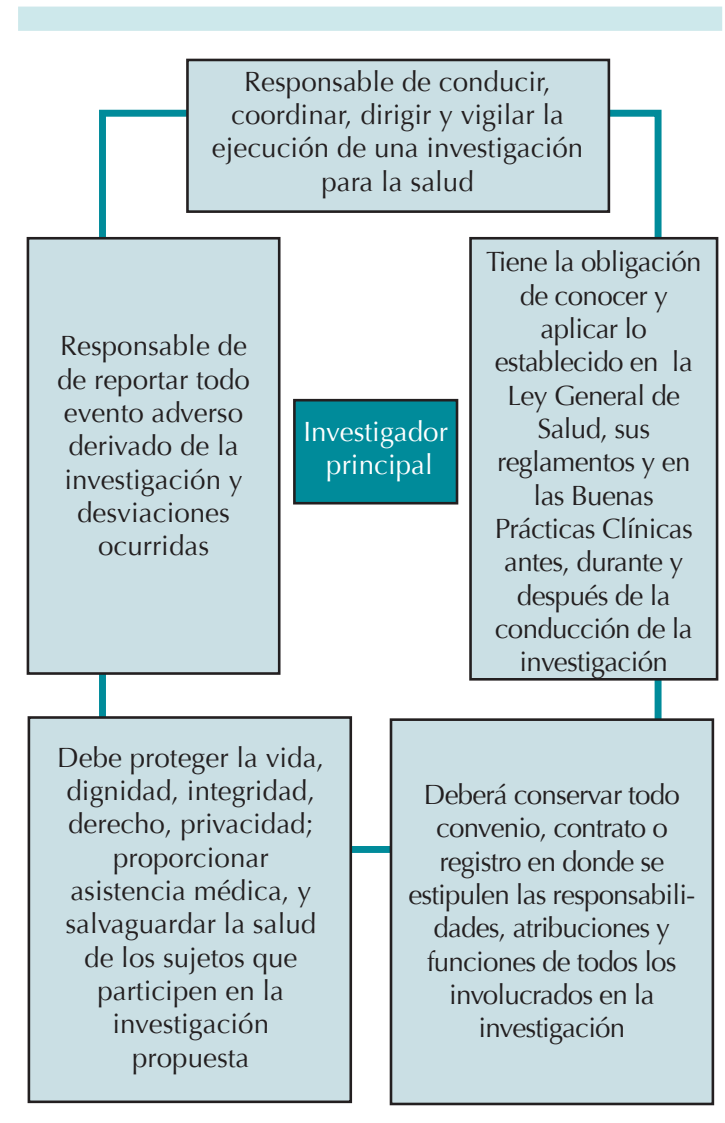

Figura 2. Resumen de las responsabilidades del investigador principal al conducir un estudio clínico. vestigación del protocolo y debe proporcionarle una copia del folleto del investigador, así como de todos los documentos sujetos a revisión. ${ }^{20}$

De acuerdo al artículo 41 Bis de la Ley General de Salud define "Comité de Ética en Investigación" como el responsable de evaluar y dictaminar los protocolos de investigación en seres humanos y muestras biológicas de seres humanos, atendiendo las recomendaciones de carácter ético que correspondan, así como de elaborar lineamientos y guías éticas institucionales para la investigación en salud, debiendo dar seguimiento a sus recomendaciones. El Comité de Ética en Investigación deberá funcionar de acuerdo a lo establecido en la Ley General de Salud, el Reglamento de la Ley General de Salud en Materia de Investigación para la Salud (LGSMIS), el Reglamento de la Ley General de Salud en Materia de Prestación de Servicios de Atención Médica (RLGSMPSAM), las disposiciones, lineamientos y guías emitidas por la Comisión Nacional de Bioética (CNB o CONBIOETICA), lo establecido en las Buenas Prácticas Clínicas (ICH-E6-R1) y demás disposiciones aplicables; el Comité de Ética en Investigación deberá registrarse ante la COFEPRIS mediante el trámite denominado "Registro de Comisiones de Investigación, Ética y Bioseguridad" habiendo cumplido previamente con el proceso de autoevaluación ante la Comisión Nacional de Bioética; una vez cumplidos los requisitos la Comisión Nacional de Bioética emitirá una constancia de registro en un máximo de diez días hábiles.

El Comité de Ética en Investigación debe establecer e implementar una "política general sobre la confidencialidad de la información" que reciben y evalúan, para los miembros del Comité, tal y como lo establece la "Guía Nacional para la Integración y el funcionamiento de los Comités de Ética en Investigación", emitida por la Comisión Nacional de Bioética, debe establecer e implementar un "código de conducta" para los miembros del comité, debe establecer 
e implementar su "Manual de Procedimientos Operativos", donde se establezcan todas y cada una de las actividades que lleva a cabo el Comité de Ética en Investigación, así como un listado actualizado de los integrantes que lo compongan; estos procedimientos así como la lista de integrantes deberán ser remitidos a la Comisión Nacional de Bioética y podrán ser solicitados por el investigador, el patrocinador y/o las autoridades regulatorias..$^{20,21}$

La documentación necesaria para la solicitud de autorización de un protocolo de investigación en seres humanos a COFEPRIS debe de cumplir con las siguientes características:

Estar en idioma español, si es necesario anexar traducción del documento; todas y cada una de las hojas deben de estar foliadas; la información deberá estar unida por un sistema de broches o cintas, permitiendo la libre apertura; la separación entre documentos debe ser mediante separadores del color. Cada trámite deberá de contar con la clasificación adecuada mediante una homoclave, nombre del trámite, modalidad y descripción, según corresponda ${ }^{20,22}$ (Cuadro 1).

En cuanto a los recursos y responsabilidades, el investigador principal debe tener tiempo suficiente para dirigir, supervisar y culminar el estudio; debe contar con la cantidad suficiente de personal calificado e instalaciones de acuerdo al diseño del estudio, el número de sujetos a incluir y dar seguimiento al cronograma de actividades del estudio; asegurarse que todos los involucrados en el estudio estén entrenados de manera adecuada. Se debe contar con el asesoramiento de un médico calificado para aprobar o no las decisiones de seguridad de los sujetos participantes en el estudio; contar con atención médica para cualquier evento adverso e informar al médico de cabecera del sujeto sobre su participación en el estudio y si está de acuerdo.
Cuadro 1. Documentación necesaria para la solicitud de autorización de COFEPRIS de un protocolo de investigación

La documentación que debe someterse es la siguiente: ${ }^{22}$ - Comprobante de pago de derechos, en términos de la Ley Federal de Derechos

- Autorización de funcionamiento del usuario: Licencia sanitaria o aviso de funcionamiento de responsable sanitario

- Autorización de funcionamiento del centro de investigación:

Licencia sanitaria o aviso de funcionamiento

- Comité de ética en investigación que aprobó el protocolo:

Comprobante de registro ante la SSA

Dictamen favorable de la Comisión Nacional de Bioética Dictamen favorable emitido por el Comité de Ética en Investigación para la conducción del estudio clínico, una vez revisado el protocolo de investigación, carta de consentimiento informado, cuando aplique asentimiento informado

Manual del investigador, información para prescribir o documento equivalente, encuestas, cuestionarios, índices o escalas validadas, tarjeta del paciente, diario del paciente, información para el sujeto (manual del usuario, folleto del producto, instrucciones de uso del producto, etc.)

- Autorización del titular del centro de investigación

- Carta descriptiva de los recursos del centro

- Carta descriptiva de los recursos para atención de urgencias o convenio hospitalario para cubrir urgencias

- Carta de aceptación, confidencialidad y compromiso de reporte de sospechas de reacciones y eventos adversos firmada por el investigador principal

- Currículo vitae y cédula profesional del investigador principal, versión en electrónico

- Preparación académica y experiencia del personal médico, paramédico y otros expertos que participarán en las actividades de la investigación

- Cronograma del estudio

- Cantidad aproximada de insumos de importación que se requieren en cada etapa del estudio (información que no se incluirá en el oficio de autorización)

Toda investigación en seres humanos deberá realizarse en una institución o establecimiento, el cual deberá contar con la infraestructura y capacidad resolutiva suficiente, para proporcionar la atención médica adecuada o en su caso, a través de terceros, ante la presencia de cualquier efecto adverso de la maniobra experimental expresada en el proyecto o protocolo de investigación autorizado. Debe contar con un servicio para la atención de urgencias médicas. En su caso, 
se deberá contar con un convenio suscrito con un establecimiento para la atención médica de mayor capacidad resolutiva, que, a manera de tercero, brinde dicha atención de urgencias. ${ }^{20}$

El centro de investigación deberá ingresar aviso de funcionamiento, así como presentar aviso de designación de Responsable Sanitario ante COFEPRIS en seguimiento a la Ley General de Salud y el reglamento de insumos para la salud. ${ }^{23}$

Compromisos del investigador principal una vez obtenida la aprobación para conducir un estudio clínico

El investigador principal tiene el compromiso de registrarse en las siguientes plataformas de registros electrónicos, una vez que obtiene la aprobación para conducir estudios clínicos. EI Registro Nacional de Estudios Clínicos (RNEC), que es una base de datos electrónica establecida en 2013 por la Secretaría de Salud de México a través de la COFEPRIS y la Comisión de Autorización Sanitaria, para informar de manera general a toda la comunidad (médica y no médica) sobre los estudios clínicos que involucran seres humanos y que se conducen y fueron sometidos a COFEPRIS. Este sitio está disponible al público en general a través de la página web de la COFEPRIS. La obligatoriedad de este registro está referida en el "Oficio de Autorización" que emite COFEPRIS a los estudios sometidos a su aprobación y se tiene un plazo no mayor a 5 días hábiles para que se ingrese la información complementaria en el Registro Nacional de Estudios Clínicos, a partir de la recepción de dicho oficio. ${ }^{24}$

La plataforma de registros internacionales de estudios clínicos "ICTRP" de la OMS tiene la finalidad de garantizar que quienes participan en la toma de decisiones en el área de la atención de la salud tengan acceso a una visión completa de la investigación. Es un recurso para mejorar la transparencia de la investigación y, fundamentalmente, consolida la validez y el valor de los datos científicos. Esta información se publica en un sitio web de acceso público.

El registro de todos los estudios clínicos de intervención es una responsabilidad científica, ética y moral. ${ }^{25}$

El sitio web de acceso público llamado "ClinicalTrials.gov", creado por Los Institutos Nacionales de la Salud (NIH por sus siglas en inglés) y la Administración de Alimentos y Medicamentos de los Estados Unidos (FDA por sus siglas en inglés), es un registro de información de estudios clínicos para probar la efectividad de medicamentos en experimentación para enfermedades o condiciones serias o que atentan la vida; este registro es proporcionado y actualizado por los patrocinadores o investigadores principales que conducen los estudios clínicos. ${ }^{26}$

La base de datos sobre estudios clínicos "EudraCT" de la Agencia Europea de Medicamentos (EMA) hace públicos a través del Registro de Estudios Clínicos de la Unión Europea (EUCTR) los resúmenes de resultados de estudios clínicos (Figura 3).

Productos que se obtienen al participar en la
conducción de estudios clínicos

Contribución en la búsqueda de mejores formas de prevenir, detectar y tratar enfermedades $y$ afecciones con impacto en la salud; fomento al desarrollo científico y tecnológico dando a conocer los resultados con importancia médica a través de publicaciones de artículos en revistas, debates o reuniones médicas y científicas y por medios de comunicación; estímulo al funcionamiento de establecimientos públicos y privados destinados a la investigación y a la formación de personal para la salud en materia de investigación en seres humanos. 


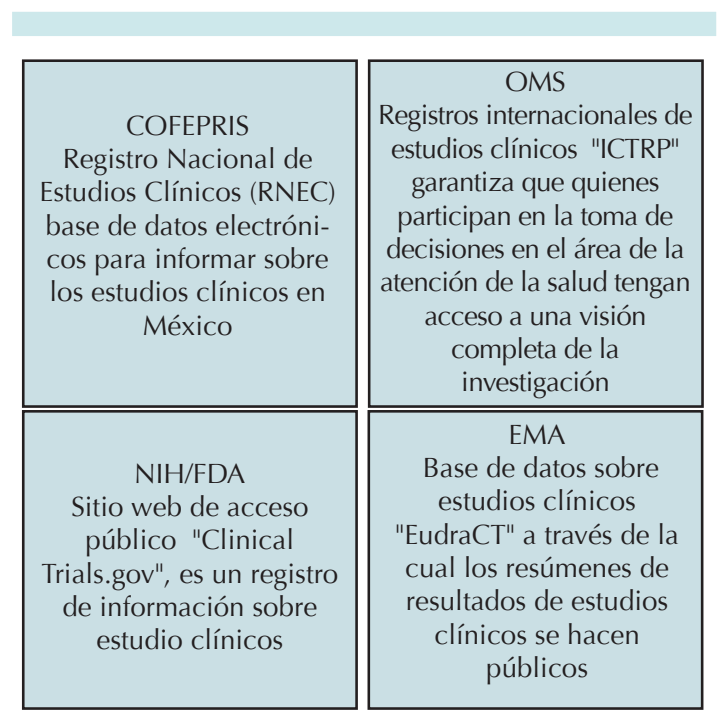

Figura 3. Plataformas de registros electrónicos nacionales e internacionales de estudios clínicos de acceso al público.

\section{CONCLUSIÓN}

El centro de investigación o el investigador principal con interés en ser seleccionado como candidato para participar en estudios clínicos en cualquier fase de investigación deberán tener un claro entendimiento y cumplir con todos los lineamientos y requisitos claramente definidos en los principios científicos, éticos y regulatorios nacionales e internacionales referidos en este artículo.

\section{REFERENCIAS}

1. Código de Nüremberg (1947).

2. Declaration of Helsinki. 18th World Medical Assembly, Helsinki, Finland (June1964).https://es.wikipedia.org/wiki/ Declaración_de_Helsinki

3. 29th World Medical Assembly, Tokyo, Japan (October 1975). www.cirp.org/library/ethics/tokyo

4. 35th World Medical Assembly, Venice, Italy (October 1983). www.wma.net/en/30publications/10policies/b3/

5. 41st World Medical Assembly, Hong Kong (September 1989) www.who.int/bulletin/archives/79(4)373.pdf.
6. 48th World Medical Assembly, Somerset, South Africa (October 1996). www.up.ac.za/.../declarationofhelsinki_fortaleza_bra...

7. 52nd World Medical Assembly, EdimburgH, Scotland (October 2000).www.wma.net/en/30publications/10policies/b3/

8. Pautas éticas internacionales para la investigación biomédica en seres humanos. Preparadas por el Consejo de Organizaciones Internacionales de las Ciencias Médicas (CIOMS) en colaboración con la Organización Mundial de la Salud. Ginebra 2002. http://www.cioms.ch/; http://www.who.int/es/.

9. Buenas Prácticas Clínicas: Documento de las Américas. IV Conferencia Panamericana para la armonización de la reglamentación farmacéutica. RepúblicaDominicana: Conferencia; 2005.

10. $\mathrm{ICH}$ Harmonized Tripartite Guideline. Guideline for good clinical practice. May 1st 1996.

11. Ley General de Salud. Última reforma publicada DOF 0406-2014.

12. Reglamento de la Ley General de Salud en Materia de Investigación para la Salud. Publicado en el Diario Oficial de la Federación el día 06 de enero de 1987.

13. Reglamento de la Comisión Federal para la Protección contra Riesgos Sanitarios. Publicado en el Diario Oficial de la Nación el día martes 13 de abril de 2004.

14. www.cofepris.gob.mx/

15. Lineamientos BPC 31052012 COFEPRIS.

16. Norma Oficial Mexica NOM-220-SSA1-2002, Instalaciones y Operación de la Farmacovigilancia.

17. Norma Oficial Mexicana NOM-168-SSA1-1998, del expediente clínico* Publicada en el Diario Oficial de la Federación, el 30 de septiembre de 1999.

18. Estructura Expediente para Protocolos Clínicos. Cerón Sánchez, Areli. Comisión de Autorización Sanitaria.

19. Norma Oficial Mexicana NOM-004-SSA3-2012, Del expediente clínico.

20. Norma Oficial Mexicana NOM-012-SSA3-2012, Que establece los criterios para la ejecución de proyectos de investigación para la salud en seres humanos.

21. http://www.conbioetica-mexico.salud.gob.mx

22. Guía para sometimiento de protocolos nuevos o iniciales. COFEPRIS.

23. Marco Jurídico. Establecimientos y Productos Biológicos. http:// www.cofepris.gob.mx/AS/Paginas/Establecimientos\%20y\%20 productos\%20biologicos/AvisoFuncionamiento.aspx

24. Manual de usuario de Registro Nacional de Estudios Clínicos (RNEC).COFEPRIS. http://www.cofepris.gob.mx/AS/ Paginas/Estudios\%20Clínicos/Registro\%20Nacional\%20 de\%20Estudios\%20Clínicos\%20(RNEC)/Registro-Nacionalde-Estudios-Clínicos-(RNEC).aspx

25. International Clinical Trials Registry Platform (ICTRP). WHO http://www.who.int/ictrp/en/

26. http://clinicaltrials.gov/https://eudract.ema.europa.eu/ 